J. Lake Sci.(湖泊科学), 2017, 29(2): 326-333

DOI 10. 18307/2017. 0208

(c) 2017 by Journal of Lake Sciences

\title{
鄱阳湖沉积物和水界面磷的交换通量”
}

\author{
向速林 ${ }^{1,2}$, 陶术平 ${ }^{1}$, 吴代赦 ${ }^{2}$ \\ ( 1 : 华东交通大学环境工程系, 南昌 330013 ) \\ (2: 南昌大学教育部鄱阳湖湖泊生态与生物资源利用重点实验室, 南昌 330047)
}

\begin{abstract}
摘 要: 采用扩散模型法与实验培养法对鄱阳湖沉积物和水界面间可溶性总磷和可溶性磷酸盐的界面交换过程进行研 究, 并探讨了其影响因素. 结果表明, 利用 2 种方法得到鄱阳湖各站点可溶性总磷和可溶性磷酸盐在沉积物与水界面间 的交换方向不完全相同, 大部分站点沉积物是磷的源, 其中, 利用扩散模型法估算的可溶性总磷和可溶性磷酸盐平均扩 散通量分别为 0.052 和 $0.047 \mathrm{mg} /\left(\mathrm{m}^{2} \cdot \mathrm{d}\right)$, 而实验培养法测得可溶性总磷和可溶性磷酸盐的平均交换通量则分别为 0.25 和 $0.24 \mathrm{mg} /\left(\mathrm{m}^{2} \cdot \mathrm{d}\right)$, 且各站点利用扩散模型法测得磷的交换通量均小于实验培养法的计算结果. 此外, 上覆水溶解氧浓 度及水体温度对可溶性总磷和可溶性磷酸盐的交换过程均具有一定的影响, 表现为温度越高, 溶解氧浓度越小, 可溶性 总磷和可溶性磷酸盐的交换越强烈.
\end{abstract}

关键词: 鄱阳湖; 磷; 沉积物和水界面; 交换通量

\section{Exchange fluxes of phosphorus at the sediment-water interface in Lake Poyang}

\author{
XIANG Sulin ${ }^{1,2}$, TAO Shuping ${ }^{1} \&$ WU Daishe ${ }^{2}$ \\ (1: Department of Environment Engineering, East China Jiaotong University, Nanchang 330013, P.R.China) \\ (2: Key Laboratory of Lake Poyang Ecology and Bioresource Utilization of MOE, Nanchang University, Nanchang 330047, \\ P.R.China)
}

\begin{abstract}
Through diffusive model methods and laboratory incubation experiments, exchanging fluxes of dissolved total phosphorus (DTP) and dissolved phosphate(DIP) at the sediment-water interface of the Lake Poyang were measured, and their influencing factors were identified. The results show that the exchanging direction of DTP and DIP were unascertainable by applying the two methods, and the sediment is a source of phosphorus in most locations. The average diffusive fluxes of DTP and DIP at the sediment-water interface using diffusive model methods were 0.052 and $0.047 \mathrm{mg} /\left(\mathrm{m}^{2} \cdot \mathrm{d}\right)$, respectively. The exchanging fluxes through the laboratory incubation experiments were higher than that using the diffusive model methods for all sites, and the average exchanging fluxes of DTP and DIP at the sediment-water interface were 0.25 and $0.24 \mathrm{mg} /\left(\mathrm{m}^{2} \cdot \mathrm{d}\right)$, respectively. In addition, the fluxes of DTP and DIP had superior differences under different contents of dissolved oxygen and different temperatures. The exchange of DTP and DIP increased with the increasing temperature and decreasing dissolved oxygen. The results are useful for the eutrophication control and ecological restoration in the Lake Poyang.
\end{abstract}

Keywords: Lake Poyang; phosphorus; sediment-water interface; exchanging flux

湖泊富营养化已成为当前主要的水环境问题, 沉积物则是湖泊氮、磷营养盐的重要储存库, 同时也是湖 泊水体营养盐的内负荷, 沉积物与水界面是环境中水相和沉积相之间的转换区 ${ }^{[1]}$, 而沉积物与水界面营养 盐的交换与迁移扩散过程是决定湖泊营养状态的重要参数, 对上覆水体的水质及湖泊富营养化均具有重要 的影响 ${ }^{[2]}$. 沉积物与水界面是沉积物和上覆水体之间进行物质交换以及营养物质进行物理、化学和生物作 用的重要场所, 是物质地球化学循环的耦合区和生物的主要栖息地带 ${ }^{[3]}$, 其在沉积物与上覆水体营养物质 输送和交换方面均具有重要作用. 氮、磷等营养盐在沉积物与水界面的迁移扩散是水生生态系统营养盐循

* 江西省教育厅科技项目 (GJJ150540) 和江西省自然科学基金项目 (20114BAB213020) 联合资助. 2016-03-17 收 稿;2016-07-10 收修改稿.向速林(1978 ), 男,博士, 副教授;E-mail: slxiang2001@ 163.com. 
环的重要过程,包含复杂的生物地球化学过程, 且受到界面处沉积物组成、沉积物中氮磷含量以及存在形 态、环境因子、底栖生物与微生物和水动力条件等因素的影响 ${ }^{[4]}$. 营养盐在沉积物与水界面的交换过程同时 受多种因素的影响,交换量的变化取决于这些因素的共同作用 ${ }^{[5-6]}$. 国内外有关沉积物与水界面营养盐的交 换通量研究开展的相对较多, 其中又以河口及海湾地区为主 ${ }^{[7-10]}$, 同时亦有探讨生物扰动、环境条件等因素 对湖泊与河流沉积物与水界面营养盐交换影响的研究 ${ }^{[11-12]}$. 但由于水域特点、环境特点不同, 研究结果不尽 相同, 反映了区域特点带来界面交换特征的差异. 故而, 探讨不同区域特点的湖泊沉积物与水界面营养盐的 交换过程对掌握营养物质的循环动力学和湖泊水体富营养化的内在机理具有重要意义.

鄱阳湖位于江西省北部, 长江中下游南岸, 是中国的第一大淡水湖, 是一个过水性、吞吐型、季节性大型 浅水湖泊 ${ }^{[13]}$. 随着社会经济的迅速发展, 农药和化肥的大量施用,城镇排污量和人口增加使大量的营养物 质不断地流入湖泊, 鄱阳湖富营养化程度日渐增加, 严重制约了区域经济的可持续发展. 其中, 沉积物对鄱 阳湖水体中营养物质的收支和营养物质的循环动力学以及水体富营养化都具有重要作用, 而界面营养盐交 换对湖泊水体中营养物质含量的影响较大. 因此, 研究鄱阳湖沉积物与水界面间营养盐的交换特征对了解 营养盐在沉积物与水界面的迁移转化过程、认识鄱阳湖富营养化进程及控制水体富营养化具有重要的理论 和实践意义. 目前有关鄱阳湖营养盐的研究主要集中在水体中营养盐浓度及其时空分布特征, 而尚少见到 有关沉积物与水界面间营养盐交换通量的研究. 本研究主要采用实验培养法及扩散模型法对鄱阳湖沉积物 与水界面营养盐磷的交换通量进行研究, 并对 2 种方法的结果进行对比分析, 同时探讨了上覆水体中溶解 氧浓度及温度变化对实验培养过程中磷交换通量的影响, 以期为掌握鄱阳湖的内源磷负荷提供参考依据.

\section{1 材料与方法}

\section{1 样品采集}

鄱阳湖柱状沉积物样品分别采集于赣江、修水及饶河河口区的康山 (L1)、龙口 (L2)、南矶 (L3) 与吴城 (L4) 站点, 以及湖区的都昌 (L5) 和星子 ( L6) 站点 (图 1). 分别在每个站点利用柱状沉积物采样器 (内径 50 $\mathrm{mm}$ ) 采集厚度约为 $30 \mathrm{~cm}$ 的若干柱状样品, 其中, 部分样品用来测定沉积物含水率及沉积物间隙水中磷浓 度, 剩余样品用于室内模拟培养实验, 这部分柱状样品需将现场采集的上覆水小心加人柱状采样器内, 尽可 能减少对底部沉积物的扰动. 同时采集现场上覆水样, 并现场测定上覆水的温度及溶解氧浓度. 此外, 用来 测定间隙水中磷浓度的沉积物样品 (表层 $0 \sim 2 \mathrm{~cm}$ 样品) 在 $4 \mathrm{~h}$ 之内以 4000 转 $/ \mathrm{min}$ 的转速离心 $30 \mathrm{~min}$, 离心 后吸出上层液体, 并用孔径为 $0.45 \mu \mathrm{m}$ 的尼龙膜过滤后加人 $\mathrm{HgCl}_{2}$ 固定在 $4{ }^{\circ} \mathrm{C}$ 下保存待用, 用来测定间隙水 中可溶性总磷 (DTP) 和可溶性磷酸盐 (DIP) 浓度.

\section{2 研究方法}

研究磷在沉积物与水界面的交换过程主要有实验培养法、现场测定法、扩散模型法以及质量平衡法等 方法. 本研究主要采用实验培养法及扩散模型法研究鄱阳湖沉积物与水界面磷的交换通量.

扩散模型法是通过实验测定沉积物间隙水和上覆水中磷浓度, 利用 Fick 第一定律计算界面间的扩散通 量, 其扩散通量计算公式为:

$$
F=-\phi \cdot D_{s} \frac{\partial C}{\partial Z}
$$

式中, $\phi$ 为沉积物孔隙度, 可以根据沉积物中含水率计算确定 ${ }^{[14]} . D_{\mathrm{s}}$ 为沉积物扩散系数,一般根据自由状态 下 $D_{0}$ 来确定, 其中 $D_{0}$ 为只与溶质溶液有关的、在无限稀释条件下的扩散系数, 根据文献 ${ }^{[15]}$, 其值为 $D_{0}=7.0 \times$ $10^{-6} \mathrm{~cm}^{2} / \mathrm{s}$, 从而计算沉积物中 DTP 和 DIP 的扩散系数 $D_{\mathrm{s}}$. 而 $\partial C / \partial Z$ 为单位距离内 DTP 和 DIP 浓度梯度, 其 计算方法是利用沉积物与水界面 $2 \mathrm{~cm}$ 深的沉积物间隙水与上覆水的磷浓度差计算得到, 即浓度梯度为 $0 \sim 2$ $\mathrm{cm}$ 沉积物间隙水中磷浓度与底层湖水浓度差除以 $1 \mathrm{~cm}$ 得到.

实验培养法是将采集的沉积物置于培养箱中, 均衡 $24 \mathrm{~h}$ 后, 按 $0 、 6 、 12 、 24 、 48 \mathrm{~h}$ 时间间隔,在距沉积物表 面约 $5 \mathrm{~cm}$ 处, 利用注射器吸取 $50 \mathrm{ml}$ 管内上覆水, 并利用 $0.45 \mu \mathrm{m}$ 微孔膜过滤, 而后置于 $50 \mathrm{ml}$ 塑料瓶中, 加 人 2 滴饱和 $\mathrm{HgCl}_{2}$ 溶液固定后在 $4{ }^{\circ} \mathrm{C}$ 保存, 用来测定上覆水样中 DTP 和 DIP 浓度. 实验培养过程主要有 2 个 部分: (1) 按实测现场上覆水体溶解氧浓度及水温进行培养实验, 测定与现场条件更接近的磷交换通量; 
(2) 在上述实验基础上改变培养的上覆水溶解氧浓度及水温, 即测定不同水温及溶解氧浓度下的磷交换通 量, 分析溶解氧浓度及水温的变化对交换通量的影响. 其中, 上覆水中各项参数按如下条件进行控制: 1) 上 覆水溶解氧浓度控制: 持续曝气充氧, 用溶氧仪测定上覆水溶解氧浓度, 达平衡时溶解氧 $>8.2 \mathrm{mg} / \mathrm{L}$, 即为饱 和状态; 持续曝气, 并控制充氧量, 维持上覆水中溶解氧浓度在 $5 \sim 7 \mathrm{mg} / \mathrm{L}$ 间, 即为好氧状态; 间断曝气充氧, 维持上覆水溶解氧浓度在 $2 \sim 4 \mathrm{mg} / \mathrm{L}$ 之间, 即为缺氧状态; 在相同装置中充人高纯 $\mathrm{N}_{2}$, 使上覆水处于厌氧状 态, 上覆水中溶解氧浓度 $<1.0 \mathrm{mg} / \mathrm{L}$, 即为厌氧状态. 充气以后立即用橡皮塞将瓶密封, 置于暗处, 放于 $20^{\circ} \mathrm{C}$ 培养箱培养. 2) 上覆水温度的控制: 利用恒温水域振荡器进行上覆水温度控制, 使上覆水温度分别控制在 $10 、 15 、 20 、 25^{\circ} \mathrm{C}$ 左右进行温度影响实验,在实验过程中定时监测和校正上覆水温度值.

沉积物与水界面 DTP 和 DIP 的交换通量根据培养实验前后水体中 DTP 和 DIP 的浓度变化直接计算获 得 ${ }^{[3,16]}$, 计算公式如下, 其中正值表示磷由沉积物进人上覆水体, 负值则相反.

$$
\begin{gathered}
F=M \cdot A^{-1} \cdot T^{-1} \\
M=\sum V_{t}\left(C_{t}-C_{t-1}\right)
\end{gathered}
$$

式中, $F$ 为营养盐交换通量 $\left(\mathrm{mg} /\left(\mathrm{m}^{2} \cdot \mathrm{d}\right)\right) ; M$ 为在 $t$ 时间内总的营养盐浓度变化量 $(\mathrm{mg}) ; V_{t}$ 为 $t$ 时刻上覆水 的总体积 $(\mathrm{L}) ; A$ 为沉积物交换表面面积 $\left(\mathrm{cm}^{2}\right) ; \mathrm{C}_{t} 、 C_{t-1}$ 分别为 $t$ 和 $t-1$ 时刻水中的磷浓度.

沉积物间隙水与上覆水中磷浓度的分析方法参照《湖泊富营养化调查规范》进行 ${ }^{[17]}$, 其 DTP 浓度采用 过硫酸钾消解法, 用光程为 $5 \mathrm{~cm}$ 吸收池, 在 $700 \mathrm{~nm}$ 处测定吸光度, 并作空白实验; DIP 浓度采用钿锑抗分光 光度法,并在 $700 \mathrm{~nm}$ 处测定其吸光度.

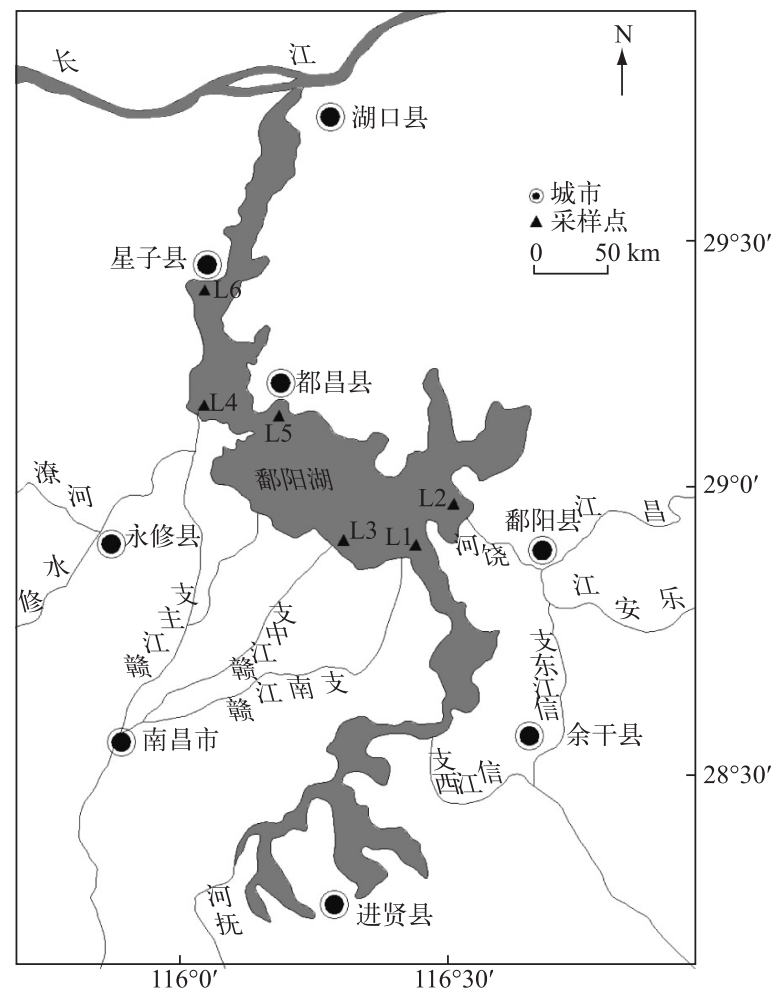

图 1 鄱阳湖采样点分布

Fig.1 The locations of sampling sites in Lake Poyang 


\section{2 结果}

\section{1 扩散模型法估算和实验培养计算结果}

利用扩散模型法估算的鄱阳湖各站点沉积物与水界面间 DTP 和 DIP 平均扩散通量分别为 $0.052 、 0.047$ $\mathrm{mg} /\left(\mathrm{m}^{2} \cdot \mathrm{d}\right)$ (图 2a). 此外, 多数站点中的 DTP 的扩散通量均略大于 DIP 的扩散通量,其中, L1、L3、L4、L5 与 L6 站点磷的扩散方向为从沉积物向上覆水扩散, 沉积物表现为上覆水中 DTP、DIP 的源; 而 L2 站点磷的 扩散方向为从上覆水向沉积物扩散, 沉积物表现为上覆水中 DTP、DIP 的汇. 空间分布上, 各站点 DTP、DIP 扩散通量具有一定的差异, 其中,站点 L5、L6 的 DTP 和 DIP 交换通量略高于其它站点,究其原因可能是站点 L5、L6 位于污染物排放区域,且该区域农业面源污染较为突出, 同时水流较小,沉积物中污染物质的积累量 同比较大. 其它站点则位于相对开阔的河口区域, 水动力扰动较大, 磷等污染物质不容易在沉积物中积累下 来, 且这些区域上游带来的农业面源污染相对较小, 故而这些区域具有更低的交换通量. 水生植物可以通过 光合作用、呼吸作用、残体分解等对沉积物表层水体或间隙水中 $\mathrm{pH} 、 \mathrm{DO}$ 等环境条件产生影响,并间接影响 间隙水与上覆水中磷浓度, 从而影响界面磷的扩散过程, 本研究中不同站点水生植物的分布情况不尽相同, 也可能是各站点磷扩散存在差异的原因之一. 此外, 鄱阳湖是季节性、过水性湖泊,水位变化较大, 水位变化 会影响上覆水特性与沉积物的含水率等理化性质,而本研究中各站点高程不同,水位变化对各站点的影响 也不同, 鄱阳湖水位的变化也可能导致各站点沉积物及间隙水中磷浓度发生不同的变化, 并进一步对界面 磷扩散造成影响,具体影响规律还需做进一步研究.

实验培养法可基本不破坏沉积物性状,并能模拟控制各种环境条件进行实验,因而得到了较广泛的应 用. 图 $2 \mathrm{~b}$ 为利用实验培养法测定的鄱阳湖各站点沉积物与水界面间 DTP、DIP 的交换通量, 其培养条件为实 测上覆水中溶解氧浓度及水体温度. 结果显示, 各站点 DTP、DIP 的平均交换通量分别为 0.25 和 0.24 $\mathrm{mg} /\left(\mathrm{m}^{2} \cdot \mathrm{d}\right)$. 实验培养法测定结果与扩散模型法计算结果相似, 实验培养法测定的鄱阳湖各站点交换通量 的空间分布特征与扩散模型法基本一致, 站点 L4 的 DTP 和 DIP 交换通量略大于其它站点, 且同样表现为多 数站点沉积物与水界面 DTP 的交换通量略大于 DIP 的交换通量, 其中, L1、L3、L4、L5 与 L6 站点磷的交换方 向为从沉积物向上覆水进行扩散, 沉积物表现为上覆水中 DTP、DIP 的源; 而 L2 站点磷的交换方向为从上覆 水体向沉积物扩散, 沉积物表现为上覆水中 DTP、DIP 的汇. 从界面交换通量的正负变化情况来看, 不同站 点存在着一定范围的正负变化, 说明鄱阳湖水体与沉积物中的磷存在一定程度的源汇转换现象.

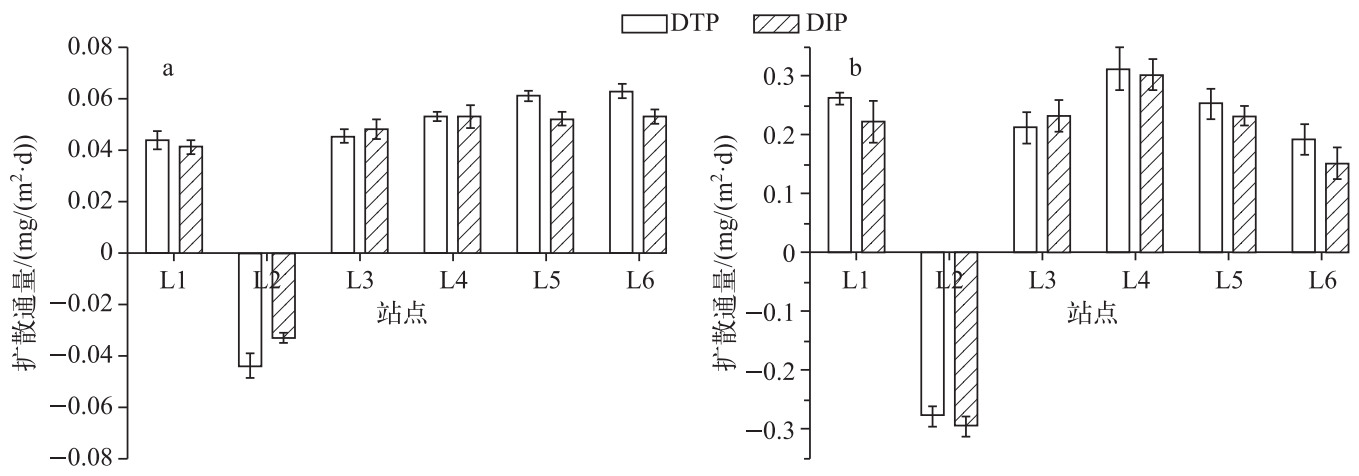

图 2 扩散模型法估算 (a) 和实验培养法计算 (b) 的沉积物与水界面间扩散通量

Fig.2 DTP and DIP diffusion fluxes of sediment and water interface by diffusive model methods(a) and laboratory incubation experiments (b)

\section{2 扩散模型法和实验培养法对比分析}

通过对 2 种方法测定结果进行对比, 鄱阳湖各站点实验培养法测定的 DTP 和 DIP 交换通量均远大于扩 散模型法的估算结果. 产生差异的原因可能是由于扩散层的存在, 实测上覆水中磷的浓度往往与界面处上 覆水中磷的实际浓度不完全一致 ${ }^{[18]}$, 且底层沉积物间隙水中磷浓度也会影响界面的扩散过程, 本研究主要 
利用表层沉积物间隙水浓度来计算界面浓度梯度, 没有考虑到底层间隙水磷浓度对界面扩散的影响, 也可 能是导致扩散通量较低的原因之一. 此外,扩散模型法并没有考虑到湖泊生态系统中生物扰动、风浪及湖流 扰动, 以及沉积物与水界面附近的沉淀/溶解、吸附/解吸、氧化还原等因素对氮、磷等营养盐在沉积物与水 界面交换过程的影响, 底栖生物通过搬运大量沉积物等对界面磷交换过程产生影响 ${ }^{[19]}$, 并与其生命活动有 关; 扩散模型法不能完全反映真实现场扩散过程, 实验培养法可以更真实还原现场, 结果更为准确, 因而导 致了扩散模型法与实验培养法分别测得的 DTP 和 DIP 交换通量结果有差异. 已有的研究结果显示, 铁氧化 物在沉积物表层的氧化层中形成一个氧化“帽”, 由于 $\mathrm{Fe}$ ( III) 能够与正磷酸盐形成不溶于水的沉淀物, 这种 氧化 “帽” 在阻止沉积物间隙水中磷酸盐向上扩散中起到重要作用, 同时也能够吸收上覆水体中的磷酸 盐 ${ }^{[20-21]}$, 故而影响了湖泊沉积物与水界面磷的扩散过程.

\section{3 讨论}

磷在湖泊沉积物与水界面间交换过程的影响因素较多 ${ }^{[9]}$, 主要包括上覆水温度与溶解氧浓度、沉积物 与上覆水磷浓度、底栖生物扰动、 $\mathrm{pH}$ 值、沉积物性质等. 本研究主要探讨了上覆水温度与溶解氧浓度对界面 交换过程的影响.

\section{1 溶解氧浓度对实验培养结果的影响}

沉积物上覆水中溶解氧浓度对营养盐在界面的交换过程具有重要影响, 可控制营养盐的交换方向. 在 不同上覆水溶解氧浓度培养条件下, 鄱阳湖各站点沉积物与水界面间 DTP 和 DIP 交换通量 (图 3) 可知, 上 覆水体中溶解氧浓度大小对 DTP 和 DIP 在界面的交换通量影响较大, 溶解氧浓度越小, DTP 和 DIP 的交换 越强烈, 即在缺氧条件比富氧条件 DTP 和 DIP 在沉积物与水界面上的交换通量更大, 即厌氧加速磷的吸附 或释放过程, 而好氧条件则抑制磷的吸附或释放. 富氧与缺氧的差异主要是影响水体的氧化还原条件, 一般 富氧时, 水体处于氧化环境, 而缺氧则处于还原环境. 当水体处于氧化环境时, DTP 和 DIP 可被吸附在含 Fe 氧化物的沉积物中而保存, $\mathrm{Fe}$ ( III) 与磷结合, 以磷酸铁形成沉积, 磷被固定, 限制了 DTP 和 DIP 向上覆水体 的扩散, 从而降低沉积物向上覆水可溶磷的释放量, 而还原条件下, 不溶性的 $\mathrm{Fe}(\mathrm{OH})_{3}$ 可变成可溶性的 $\mathrm{Fe}(\mathrm{OH})_{2}$, 使与铁结合的磷大量释放进人水体, 因此还原环境有利于 $\mathrm{PO}_{4}^{3-}-\mathrm{P}$ 的释放 ${ }^{[22]}$, 从而导致界面交换 过程更显著.

\section{2 温度对实验培养结果的影响}

研究表明, 上覆水温度的变化对沉积物与水界面磷的交换过程具有重要影响 ${ }^{[23-24]}$. 鄱阳湖各站点沉积 物与水界面间 DTP 和 DIP 在不同上覆水温度培养条件下的交换通量可知 (图 4), 上覆水温度对 DTP 和 DIP 在界面的交换通量均有较大的影响, 随着上覆水温度升高, 界面交换通量也将加大, 但当 DTP 和 DIP 的培养 温度达到 $20^{\circ} \mathrm{C}$ 以后, 其交换通量增加则不明显, 因此, 可以认为 DTP 和 DIP 的最佳培养温度在 $20^{\circ} \mathrm{C}$ 左右. 一 般认为, 温度变化, 直接或间接影响着沉积物中磷含量、有机物矿化速率等 ${ }^{[23-24]}$, 而且温度控制着磷在沉积 物与水界面上的物理、化学过程, 从而影响磷在界面的交换过程与通量, 当水体温度升高时, 沉积物吸附能 力下降, 固体颗粒表面的离子容易解吸进人间隙水和上覆水体 ${ }^{[25]}$, 所以导致交换通量增加. 此外, 水温能决 定各种物质在水体中的溶解度, 同时还影响沉积物中溶解氧的含量和微生物的活动能力, 而微生物活动对 磷的界面交换有一定影响. 温度较低时, 不利于沉积物中微生物活动, 磷酸盐不容易从有机质中释放出来, 从而影响沉积物中磷浓度, 进而影响界面交换过程. 上覆水温度升高促进了沉积物与水界面磷的交换过程, 故而在水体温度较高的春夏季节,鄱阳湖水体具有更高的富营养化风险.

\section{4 结论}

通过扩散模型法估算鄱阳湖各站点沉积物与水界面的营养盐 DTP 和 DIP 平均扩散通量分别为 0.052 和 $0.047 \mathrm{mg} /\left(\mathrm{m}^{2} \cdot \mathrm{d}\right)$. 利用实验培养法测得 DTP 和 DIP 平均交换通量分别为 0.25 和 $0.24 \mathrm{mg} /\left(\mathrm{m}^{2} \cdot \mathrm{d}\right)$, 其 相应交换通量均大于扩散模型法的估算结果. 实验培养法更能反映现场情况, 相对而言具有更高的精度. 此 外,界面交换方向不完全相同, 大部分站点沉积物是磷的源.

鄱阳湖上覆水中溶解氧浓度对 DTP 和 DIP 在界面间的交换通量影响较大, 溶解氧浓度越小, DTP 和 


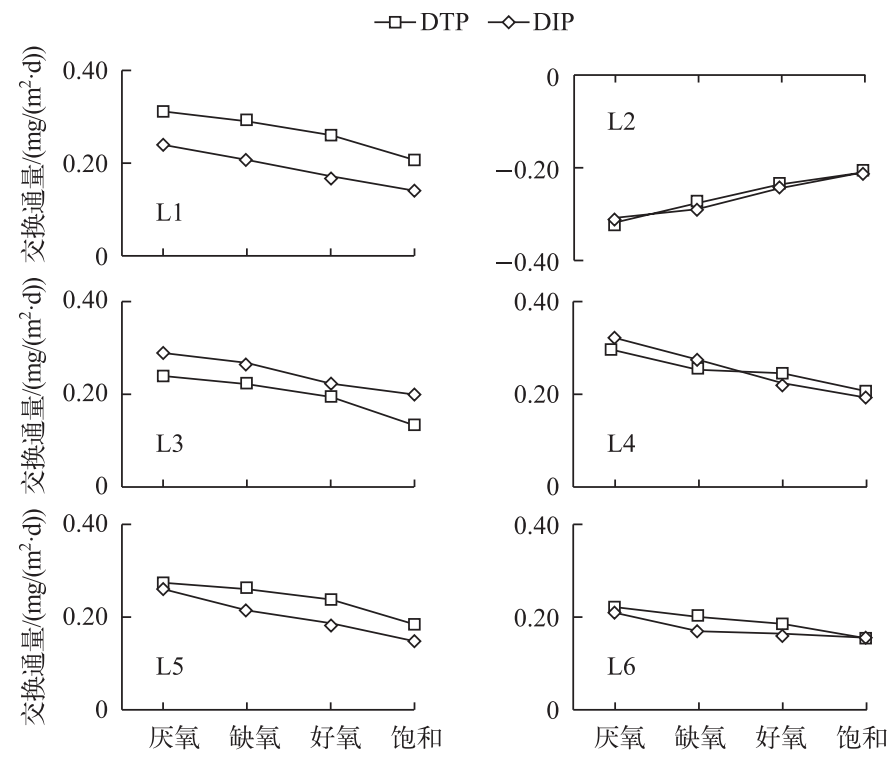

图 3 上覆水不同溶解氧浓度下 DTP 和 DIP 界面交换通量

Fig. 3The effect of different dissolved oxygen concentrations of overlying water on the DTP and DIP diffusion fluxes of sediment and water interface

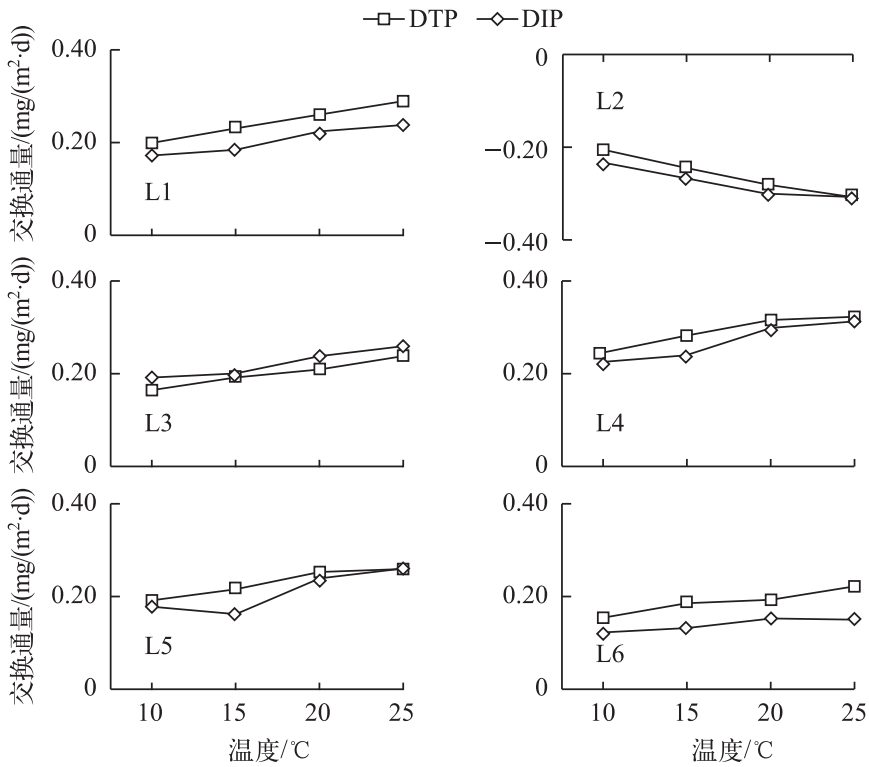

图 4 上覆水不同温度条件下 DTP 和 DIP 界面交换通量

Fig.4 The effect of different temperatures of overlying water on the DTP and DIP diffusion fluxes of sediment and water interface

DIP 的交换越强烈, 厌氧加速磷的吸附或释放过程, 而好氧条件则抑制磷的吸附或释放. 上覆水体的温度对 DTP 和 DIP 在界面间的交换通量均有较大的影响,一般温度升高时,交换通量也增加,但 DTP 和 DIP 的培养 温度达到 $20^{\circ} \mathrm{C}$ 以后, 其交换通量增加则不明显. 反映了温度较低时, 温度的变化对界面磷交换影响较大, 且 
厌氧条件更有利于磷在界面的交换过程.

\section{5 参考文献}

[ 1 ] He Jia, Chen Chunyu, Deng Weiming et al. Distribution and release characteristics of phosphorus in water-sediment interface of Lake Dianchi. J Lake Sci, 2015, 27(5) : 799-810. DOI : 10.18307/2015.0506. [何佳, 陈春瑜, 邓伟明等. 滇池 水-沉积物界面磷形态分布及潜在释放特征. 湖泊科学, 2015, 27 (5) : 799-810.]

[ 2 ] Liu Min, Hou Lijun, Xu Shiyuan et al. Nitrogen and phosphorus diffusion fluxes across sediment-water interface in estuarine and coastal tidal flats. Marine Environmental Science, 2001, 20(3): 19-24. [刘敏, 侯立军, 许世远等. 河口滨岸潮 滩沉积物-水界面 N、P 的扩散通量. 海洋环境科学, 2001, 20(3): 19-24.]

[ 3 ] Liu Jie. Research on dissolved inorganic nitrogen exchange at the sediment-water interface of the Yangtze estuarine tidal flats [Dissertation]. Shanghai: East China Normal University, 2006. [刘杰. 长江口潮滩无机氮界面交换研究 [学位论 文]. 上海: 华东师范大学, 2006. ]

[ 4 ] Gao Li, Yang Hao, Zhou Jianmin. Research progress on phosphorus release from lake sediments. Soils, 2004, 36( 1) : 1215. [ 高丽, 杨浩, 周健民. 湖泊沉积物中磷释放的研究进展. 土壤, 2004, 36 (1) : 12-15. ]

[ 5 ] Giblin AE, Weston NB, Banta GT et al. The effects of salinity on nitrogen losses from an oligohaline estuarine sediment. Estuaries and Coasts, 2010, 33(5): 1054-1068.

[ 6 ] Grandel S, Rickert D, Schluter M et al. Pore-water distribution and quantification of diffusive benthic fluxes of silicic acid, nitrate and phosphate in surface sediments of the deep Arabian Sea. Deep-Sea Research II, 2000, 47 ( 14): 2707-2734.

[ 7 ] Mozeto AA, Silverio PF, Soares A. Estimates of benthic fluxes of nutrients across the sediment-water interface ( Guarapiranga reservoir, Sao Paulo, Brazil). Science of the Total Environment, 2001, 266(1/2/3): 135-142.

[ 8 ] Jahnke RA, Nelson JR, Marinelli RL et al. Benthic flux of biogenic elements on the southeastern US continental shelf influence of pore water advective transport and benthic microalgae. Continental Shelf Research, 2000, 20: 109-127.

[ 9 ] Jiang Fenghua, Wang Xiulin, Shi Xiaoyong et al. Benthic exchange rates and fluxes of dissolved inorganic nitrogen at the sediment-water interface of Jiaozhou Bay. Marine Science, 2004, 28(4): 13-19. [蒋凤华, 王修林, 石晓勇等. 溶解无机 氮在胶州湾沉积物-海水界面上的交换速率和通量研究. 海洋科学, 2004, 28(4) : 13-19.]

[10] Wang Xiulin, Xin Yu, Shi Feng et al. The exchange rate and flux of macro-nutrients and the regulating factors at the seawater-sediment interface in the Bohai Sea. Periodical of Ocean University of China, 2007, 37(5) : 795-800. [王修林, 辛 宇, 石峰等. 溶解无机态营养盐在渤海沉积物-海水界面交换通量研究. 中国海洋大学学报, 2007, 37( 5): 795-800.]

[11] Yan Yanhong. Experimental study on the influence of Tubificid Worms' -Bioturbation on nitrogen exchanging from the sediment-water interface of Xiangjiang River [Dissertation]. Changsha: Changsha University of Science \& Technology, 2012. [间研红. 颤蚓生物扰动对湘江沉积物-水界面氮交换的影响研究 [ 学位论文]. 长沙: 长沙理工大学, 2012.]

[12] Yuan Shou. Nitrogen speciation of the sediments and flux in sediment-water interface in North Bay of Taihu Lake [Dissertation]. Handan: Hebei University of Engineering, 2015. [袁首. 太湖北部湖湾沉积物氮赋存形态及其沉积物-水界面 通量研究 [学位论文]. 邯単: 河北工程大学,2015.]

[13] Xiang Sulin, Zhou Wenbin, Nie Fahui. Phosphorus concentrations and distributions in lacustrine interstitial water of Lake Poyang. J Lake Sci, 2011, 23(6) : 868-872. DOI：10.18307/2011.0607. [ 向速林, 周文斌, 聂发辉. 鄱阳湖沉积物间 隙水中磷的含量及其分布特征. 湖泊科学, $2011,23(6): 868-872$. $]$

[14] Bolalek J, Graca B. Ammonium nitrogen at the water-sediment interface in Puck Bay(Baltic Sea). Estuarine Coastal and Shelf Science, 1996, 43: 767-779.

[15] Tu Qingying ed. Research of Chaohu Lake eutrophication. Hefei : Press of University of Science and Technology of China, 1990. [ 屠清瑛. 巢湖富营养化研究. 合肥: 中国科学技术大学出版社, 1990.]

[16] Aller RC, Mackin JE, Ullman WJ et al. Early chemical diagensis, sediment-water solute exchange, and storage of reactive organic matter near the mouth of the Changjiang, East China Sea. Continental Shelf Research, 1985, 4(1/2) : 227-251.

[17] Jin Xiangcan, Tu Qingying eds. Survey standards of lake eutrophication (2nd Edition). Beijing: China Environmental Science Press, 1990. [ 金相灿, 屠清瑛. 湖泊富营养化调查规范(第 2 版). 北京: 中国环境科学出版社, 1990.] 
[18] Liu Sumei, Jiang Wensheng, Zhang Jing. Exchange fluxes of nutrients in sediment water interface calculated by diagenetic model: A case study of Bohai. Periodical of Ocean University of China, 2005, 35(1): 145-151. [刘素美, 江文胜, 张经. 用成岩模型计算沉积物-水界面营养盐的交换通量——渤海为例. 中国海洋大学学报, 2005, 35(1): 145-151.]

[19] Zhang Jiefan, Li Qingxue, Tao Jianhua. Exchange fluxes and effect factors of nutrients between bottom sediment and water in Bohai Bay. Marine Environmental Science, 2009, 28(5): 492-496. [ 张洁帆, 李清雪, 陶建华. 渤海湾沉积物和水 界面间营养盐交换通量及影响因素. 海洋环境科学, 2009, 28(5) : 492-496. ]

[20] Jarvie HP, Mortimer RJG, Palmer-Felgate EJ et al. Measurement of soluble reactive phosphorus concentration profiles and fluxes in river-bed sediments using DET gel probes. Journal of Hydrology, 2008, 350(3/4) : 261-273.

[21] Deng Ke, Yang Shilun, Liu Sumei et al. Nutrient fluxes at sediment-water interface in the east Chongming tidal flat in winter. Journal of East China Normal University: Natural Science, 2009,(3) : 17-27. [邓可,杨世伦,刘素美等. 长江口崇 明东滩冬季沉积物水界面营养盐通量. 华东师范大学学报: 自然科学版, 2009,(3): 17-27.]

[22] Zhao Zhimei. The research of the phosphorus forms in the sediment and exchange of nutrients between the sediment-water interface in Bohai Bay [Dissertation]. Xi’an: Northwest A \& F University, 2005. [ 赵志梅. 渤海湾沉积物磷形态及营养 盐在沉积物-水界面交换的研究 [学位论文]. 西安: 西北农林科技大学, 2005.]

[23] Wang XY, Zhang LP, Zhang HS et al. Phosphorus adsorption characteristics at the sediment-water interface and relationship with sediment properties in FUSHI reservoir, China. Environmental Earth Science, 2012, 67: 146-153.

[24] Han HJ, Lu XX, Burger DF et al. Nitrogen dynamics at the sediment-water interface in a tropical reservoir. Ecological Engineering, 2014: 146-153.

[ 25] Liu Q, Liu SL, Zhao HD et al. The phosphorus speciations in the sediments up and down-stream of cascade dams along the middle Lancang River. Chemosphere, 2015, 120: 653-659. 